\title{
A METODOLOGIA UTILIZADA EM ESTUDOS QUE ENVOLVEM ECOS DA PREMATURIDADE NA HISTÓRIA DA FAMÍLIA
}

\author{
THE METODOLOGY USED IN STUDIES ABOUT \\ REPERCUSSIONS OF PREMATURITY IN THE HISTORY OF A FAMILY \\ Sylvia M. P. Pereira* \\ Maria H. C. de A. Cardoso**
}

Pereira SMP, Cardoso MHCdA. A metodologia utilizada em estudos que envolvem ecos da prematuridade na história da família. Rev Bras Cresc Desenv Hum 2005; 15(3):74-83.

Resumo: O objetivo deste artigo foi revisar a literatura para identificar a natureza da metodologia utilizada e qual a área de atuação dos autores na abordagem do impacto da prematuridade na família e, a longo prazo, na criança. Baseou-se em: bases de dados do Medline e Lilacs utilizandose como palavras-chaves: "premature, family"; "intensive care unit, neonatal"; "quality of life"; "qualitative research". Foram selecionados artigos médicos nas áreas de Pediatria, Psiquiatria, Medicina Psicossocial e nas áreas de Enfermagem e Ciências Humanas, publicados nos idiomas inglês, espanhol e português, nos últimos dez anos e artigos identificados a partir das referências bibliográficas citadas nos primeiros artigos. Utilizadas teses de pós-graduação com informações relevantes. foram revistos onze artigos que utilizaram a metodologia quantitativa com questionários estruturados e semi-estruturados, analisados por testes estatísticos de distribuição de freqüência, qui-quadrado, regressão logística e covariância e seis estudos, sendo duas teses de mestrado e quatro artigos que utilizaram a metodologia qualitativa, com entrevista não estruturada, entrevista com grupos focais, história de vida e observação participante, analisados por fenomenologia, fenomenologia-hermenêutica e análise temática. Concluiu-se que os estudos que abordam o impacto da prematuridade na família, em sua maioria, utilizam metodologia quantitativa e são produzidos na área da Medicina, principalmente Neonatologia e Psiquiatria. A metodologia qualitativa está começando a ser aplicada a esses estudos, em áreas não médicas. São necessárias novas pesquisas para que os médicos, especialmente Neonatologistas, conheçam a especificidade da metodologia qualitativa em gerar evidências, podendo ser fonte de observação tão significativa quanto aquela proveniente da pesquisa quantitativa, para estudos que abordam prematuridade e família.

Palavras-chave: Prematuridade. Família. Unidade de tratamento intensivo neonatal. Qualidade de vida. Pesquisa qualitativa.

"Geograficamente deslocados, com seu trabalho e seu modo de vida interrompido, seus ritmos biológicos transportados, desnorteados, ansiosos e terrivelmente cansados, esses pais, no delírio de suas crises, são simplesmente incapazes de entender o que

\footnotetext{
Mestre em Pediatria pela Universidade Federal do Rio de Janeiro - Instituto Fernandes Figueira. Rua Décio Vilares, 168 / 501- Copacabana. - Rio de Janeiro, RJ, Brasil CEP 22041-040 Tel e fax: (021) 2236-4498 e-mail sylvipp@ hotmail.com

** Doutora em Saúde da Mulher e da Criança pelo Instituto Fernandes Figueira-FIOCRUZ. Rua Prof. Ortz Monteiro 24/ 202 - Laranjeiras - Rio de Janeiro, RJ, Brasil CEP22245-100 Tel: (021) 2553-0052 e-mail: oscar mc@ unysis.com.br
} 
está acontecendo"Green ${ }^{1}$ (1979: 1119)

\section{INTRODUÇÃO}

A sobrevida de recém-nascidos no limite da viabilidade vem aumentando a cada déca$\mathrm{da}^{2-4}$. O interesse sobre o prognóstico destas crianças tem levado a grande número de publicações $^{5}$. A literatura sugere taxas elevadas de morbidade neurológica e de hospitalizações recorrentes na lactância, em comparação com crianças nascidas a termo ${ }^{6-8}$. Na idade escolar, outros problemas se tornam aparentes como dificuldades de comportamento e deficiências cognitivas, contribuindo para ansiedade e encargos adicionais aos pais e à família9 . Os avanços técnicos no cuidado do recém-nascido prematuro de alto risco e seu prognóstico precederam a completa investigação das implicações morais, éticas e familiares ${ }^{6}$.

Quando a gestação é interrompida pelo nascimento prematuro, os pais do recém-nascido vivem uma situação de crise. Convivem com uma "reação de luto" pelo filho perfeito que não tiveram e necessitam tempo e apoio para que possam aceitar seu filho real ${ }^{10}$. Seus sentimentos são de revolta, culpa, angústia, tristeza, medo e esperança $a^{11-12}$. A rede familiar recebe o impacto e tenta se adaptar desenvolvendo estratégias dentro e fora de seu ambiente, para lidar com a nova situação ${ }^{13}$.

A maioria dos pais de recém-nascidos internados em Unidades de Tratamento Intensivo Neonatal sofre estresse emocional ${ }^{14}$. É necessário entender o que se passa com eles. Os intensivistas devem ter uma atitude não julgadora, entender as preocupações, o comportamento dos pais e os sentimentos encobertos da família ${ }^{15}$.

A alta do recém-nascido para casa pode ser causa de ansiedade para as famílias ${ }^{16}$. Buscam ajuda entre seus pares e dividem tarefas do cuidar, as preocupações e as ansiedades. As relações com o pai e os irmãos, nos primei- ros anos, são a base do desenvolvimento da personalidade e da saúde mental. Neste período, o bebê deve ter a vivência de uma relação calorosa, íntima e contínua com a mãe ou substituta, em que ambos encontrem satisfação e $\operatorname{prazer}^{17}$. Não há clareza sobre quais são os componentes para a alta hospitalar precoce e bem sucedida, como preparar os pais para parentalidade prematura e a que ponto os intensivistas estão sobrecarregando estes pais com responsabilidades que podem comprometer sua competência em serem pais comuns. ${ }^{18}$

A maioria dos trabalhos sobre a experiência dos pais com um filho prematuro extremo, em Neonatologia, é de natureza quantitativa. ${ }^{3,5,6,19}$. Tem sido crescente a contribuição das Ciências Sociais, através da pesquisa qualitativa, para investigação na área da saúde ${ }^{20}$. As relações e o dinamismo da vida social, a atividade criadora e suas contradições integram um lado não perceptível e não captável em equações, médias e estatísticas, no processo do conhecimento ${ }^{21}$. Este estudo tem como objetivo revisar a literatura para identificar a natureza da metodologia utilizada e qual a área de atuação dos autores na abordagem do impacto da prematuridade na família e, a longo prazo, na criança.

\section{Os estudos}

Foram revistos onze artigos que avaliaram o impacto do nascimento de um recémnascido prematuro extremo na família e o impacto sobre si mesmo, após a alta hospitalar, utilizando metodologia quantitativa, com questionários estruturados e semi-estruturados, analisados por testes estatísticos de distribuição de frequiência, qui-quadrado, regressão logística e covariância (tabela 1). Foram revistos outros seis estudos, sendo duas teses de mestrado brasileiras e quatro artigos estrangeiros que utilizaram metodologia qualitativa, com entrevista não estruturada, entrevista com grupos focais, his- 
Tabela 1 - Trabalhos que utilizam metodologia quantitativa

\begin{tabular}{|c|c|}
\hline $\begin{array}{l}\text { Autor; Ano de publicação; } \\
\text { Área do conhecimento }\end{array}$ & Conclusões \\
\hline $\begin{array}{l}\text { Streiner et } \mathrm{al}^{22}(2001) \\
\text { Psiquiatria }\end{array}$ & $\begin{array}{l}\text { A decisão de intervir para salvar o recém-nascido prematuro deve } \\
\text { envolver o conhecimento do médico e o desejo dos pais. }\end{array}$ \\
\hline $\begin{array}{l}\text { Holditch et } \mathrm{al}^{23}(2003) \\
\text { Enfermagem }\end{array}$ & $\begin{array}{l}\text { As mães revivem a experiência da internação de seus recém- } \\
\text { nascido prematuro, ainda após } 6 \text { meses. }\end{array}$ \\
\hline $\begin{array}{l}\text { Tommiska et } \text { al }^{24}(2002) \text { Pediatria; } \\
\text { Medicina do Adolescente }\end{array}$ & $\begin{array}{l}\text { O nascimento de recém-nascidos extremo baixo peso é } \\
\text { "estressante" para os pais; a maioria se recupera bem após } 2 \text { anos. }\end{array}$ \\
\hline $\begin{array}{l}\text { Singer et } \mathrm{al}^{25}(1999) \\
\text { Neonatologia }\end{array}$ & $\begin{array}{l}\text { As mães apresentam reações de crise ao nascimento de filhos } \\
\text { prematuros e, após } 3 \text { anos de vida, recuperam-se bem. }\end{array}$ \\
\hline $\begin{array}{l}\text { Pierrehumbert et } \mathrm{al}^{26}(2003) \\
\text { Psiquiatria }\end{array}$ & $\begin{array}{l}\text { A reação dos pais ao nascimento prematuro media os riscos de } \\
\text { efeitos adversos tardios na criança. Devem ser instituídas medidas } \\
\text { preventivas. }\end{array}$ \\
\hline $\begin{array}{l}\text { Rivers et } \mathrm{al}^{6}(1987) \\
\text { Pediatria }\end{array}$ & $\begin{array}{l}\text { Ocorre o impacto da prematuridade na família: no relacionamento } \\
\text { com a criança, nos planos para seu futuro e na vida pessoal dos } \\
\text { pais que, apesar dos problemas, são gratos pela vida dos filhos e } \\
\text { pelo tratamento neonatal. }\end{array}$ \\
\hline $\begin{array}{l}\text { Hille et } \mathrm{al}^{27}(2001) \\
\text { Medicina Preventiva }\end{array}$ & $\begin{array}{l}\text { Recém-nascidos prematuros com diferenças culturais apresentam } \\
\text { problemas de comportamento, dificuldades de atenção e problemas } \\
\text { de comportamento social semelhantes, sugerindo a contribuição do } \\
\text { fator biológico. }\end{array}$ \\
\hline $\begin{array}{l}\text { Saigal }^{19}(2000 a) \\
\text { Neonatologia }\end{array}$ & $\begin{array}{l}\text { Adolescentes que foram recém-nascidos prematuros têm a } \\
\text { percepção sobre sua saúde, satisfação com a vida e bem estar } \\
\text { discordante de sua saúde objetiva e suas seqüelas: aprendem sobre } \\
\text { suas dificuldades, exercem os papéis esperados, têm atitude } \\
\text { positiva perante a vida. }\end{array}$ \\
\hline $\begin{array}{l}\text { Hack et } \mathrm{al}^{9}(2002) \\
\text { Neonatologia }\end{array}$ & $\begin{array}{l}\text { Recém-nascidos prematuros apresentam coeficiente de inteligência } \\
\text { diminuído, escolaridade mais baixa e maior freqüência de doenças } \\
\text { crônicas. }\end{array}$ \\
\hline $\begin{array}{l}\text { Saigal et al }{ }^{28}(2000) \\
\text { Neonatologia }\end{array}$ & $\begin{array}{l}\text { Nos primeiros } 6 \text { meses após o nascimento do recém-nascido } \\
\text { prematuro, os pais apresentam sofrimento e preocupação pela } \\
\text { saúde física e emocional de seus filhos, com impacto em suas } \\
\text { próprias saúdes emocionais e efeitos negativos na família. Relatam } \\
\text { ter pouco tempo para si mesmos. Outros filhos são afetados } \\
\text { negativamente, talvez por menor tempo de atenção dos pais. É } \\
\text { mais difícil obter trabalho por causa do recém-nascido prematuro } \\
\text { que exige maior cota de tempo para seu cuidado. A longo prazo, os } \\
\text { pais se ajustam bem ao trabalho e à vida familiar. Apesar dos } \\
\text { efeitos negativos, os pais foram favoráveis à manutenção do } \\
\text { tratamento ativo a seus recém-nascidos. }\end{array}$ \\
\hline $\begin{array}{l}\text { Saigal et al }{ }^{29}(2002) \\
\text { Neonatologia; Psicologia }\end{array}$ & $\begin{array}{l}\text { Não há diferenças na auto-estima entre adolescentes que foram } \\
\text { recém-nascidos extremo baixo peso e adolescentes que foram } \\
\text { recém-nascidos de peso normal. }\end{array}$ \\
\hline
\end{tabular}

tória de vida e observação participante, analisados por fenomenologia, hermenêutica e análise temática. A extensão das observações é variada, alcançando adultos jovens que foram recém-nascidos prematuros extremo baixo peso $^{9}$ (tabela 2).

Streiner et al. ${ }^{22}$ (2001), área de atuação, Psiquiatria, compararam as atitudes de médicos e enfermeiras neonatais, pais de recém-nascidos de extremo baixo peso e pais de recém- 
Tabela 2 - Trabalhos que utilizam metodologia qualitativa

\begin{tabular}{|c|c|}
\hline $\begin{array}{l}\text { Autor; Ano de publicação; } \\
\text { Área do conhecimento }\end{array}$ & Conclusões \\
\hline $\begin{array}{l}\text { Lamy }^{11}(1995) \\
\text { Neonatologia }\end{array}$ & $\begin{array}{l}\text { Os pais de recém-nascidos prematuros apresentam culpa, medo e } \\
\text { negação, o que dificulta o desenvolvimento do apego; as } \\
\text { informações médicas não são bem compreendidas; os pais têm } \\
\text { suas vidas pessoal e profissional devastadas }\end{array}$ \\
\hline $\begin{array}{l}\text { Gaíva }^{32}(1997) \\
\text { Enfermagem }\end{array}$ & $\begin{array}{l}\text { O nascimento de um filho prematuro, para a família, é uma } \\
\text { experiência difícil; o cuidar do filho envolve toda a rede familiar e } \\
\text { a rede de vizinhos; a mãe assume o papel central na socialização da } \\
\text { criança. }\end{array}$ \\
\hline $\begin{array}{l}\text { Nystrom \& Axelsson }{ }^{33} \text { (2002) } \\
\text { Ciências da Saúde }\end{array}$ & $\begin{array}{l}\text { As mães de recém-nascidos prematuros, em Unidade de } \\
\text { Tratamento Intensivo Neonatal, relatam sentirem-se do "lado de } \\
\text { fora", com desespero, solidão, impotência, instabilidade } \\
\text { emocional, culpa e insegurança e, em relação ao resultado do } \\
\text { tratamento, sentem confiança, alívio e amor. }\end{array}$ \\
\hline $\begin{array}{l}\text { Bialoskurski et al }{ }^{35} \text { (1999) } \\
\text { Enfermagem }\end{array}$ & $\begin{array}{l}\text { A construção do apego entre a mãe e o recém-nascido prematuro, } \\
\text { em Unidade de Tratamento Intensivo Neonatal não foi automática } \\
\text { e os fatores envolvidos em sua construção foram: estado de saúde } \\
\text { da mãe e do recém-nascido; condições do ambiente e qualidade do } \\
\text { cuidado que o recém-nascido recebeu. }\end{array}$ \\
\hline $\begin{array}{l}\text { Cox \& Bialoskurski }{ }^{36}(2001) \\
\text { Enfermagem }\end{array}$ & $\begin{array}{l}\text { Quando a mãe e a família do recém-nascido prematuro são } \\
\text { apoiadas, através de auxílio individualizado e há comunicação } \\
\text { adequada, a formação do vínculo pode ser facilitada. }\end{array}$ \\
\hline 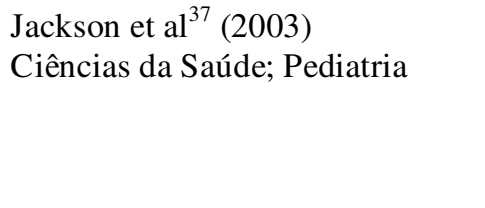 & $\begin{array}{l}\text { A parentalidade é um processo tempo-dependente, síntese da } \\
\text { experiência de alienação, confiança, responsabilidade e } \\
\text { familiaridade com o recém-nascido. Baseia-se na expectativa dos } \\
\text { pais em serem pais, na condição de saúde e no ambiente de } \\
\text { cuidado do recém-nascido. }\end{array}$ \\
\hline
\end{tabular}

nascidos de peso normal, em relação a investir no tratamento de recém-nascidos no limite da viabilidade e quem deveria estar envolvido nesta decisão. Os pais são mais favoráveis à intervenção para salvar os recém-nascidos, independente do peso de nascimento ou da condição clínica, do que os profissionais. Deve haver decisão conjunta que envolva o conhecimento do médico e o desejo dos pais. O instrumento utilizado foi questionário estruturado. A análise foi realizada através de teste estatístico de distribuição de frequiência.

Holditch et al. ${ }^{23}$, área de atuação, Enfermagem, avaliaram, à época da alta hospitalar e 6 meses após, o comportamento de mães de recém-nascidos prematuros que foram internados em Unidade de Tratamento Intensivo Neonatal. Concluíram que as mães apresentavam vivência emocional de reviver a internação, após 6 meses, semelhante à reação pós-traumática vivenciada no momento da alta. Utilizado, como instrumento, questionário semi-estruturado e feita análise estatística por distribuição de frequiência.

Tommiska et al. ${ }^{24}$, área de atuação, Pediatria, compararam estresse de pais de crianças de 2 anos de idade que foram recém-nascidos de extremo baixo peso com grupo controle de pais de crianças que foram recém-nascidos a termo. Concluíram que o nascimento de re- 
cém-nascidos extremo baixo peso é fator causal para estresse e que a maioria dos pais se recupera bem à época em que o filho atinge a idade de 2 anos. Utilizado questionário sobre estresse de pais, avaliado através de escalas numéricas.

Singer et al. ${ }^{25}$, área de atuação, Neonatologia, estudaram o nível de estresse vivenciado através do tempo, por mães cujos recémnascidos tinham diferentes graus de prematuridade e risco clínico, e de desenvolvimento. Foi realizado estudo prospectivo, longitudinal, do tipo coorte, de mães de recém-nascidos prematuros de muito baixo peso e de alto risco (portadores de broncodisplasia pulmonar) e de baixo risco e recém-nascidos a termo do grupo controle, do nascimento até a idade de 3 anos. Após 1 mês do nascimento de seus filhos, as mães dos prematuros de alto risco tinham mais estresse que as mães dos recém-nascidos de baixo risco e a termo. Após 2 anos, não havia diferença, apenas entre as mães dos recém-nascidos de baixo risco e as mães dos recém-nascidos a termo. Após 3 anos, não havia diferença entre os três grupos de mães. Concluíram que mães apresentam reações de crises ao nascimento de filhos prematuros seguidas de adaptação, em torno de $3^{\circ}$ ano de vida de seus filhos. O instrumento utilizado foi questionário com dados sobre estresse psicológico materno. A avaliação foi feita pelo teste estatístico do qui-quadrado.

Pierrehumbert et al. ${ }^{26}$, área de atuação, Psiquiatria, estudaram os efeitos das reações pós-traumáticas dos pais em problemas de sono e alimentação de crianças que foram recémnascidos de extremo baixo peso, aos 18 meses de idade. Os resultados mostraram que a gravidade dos riscos perinatais predizem, parcialmente, os problemas das crianças, sendo a intensidade das reações pós-traumáticas dos pais um importante preditor destes problemas e que devem ser instituídas medidas preventivas. Foi utilizado, como método, questionário sobre de- sordens "estressantes" pós-traumáticas perinatais. A análise foi realizada pelo teste estatístico de distribuição de frequiência.

Rivers et al. ${ }^{6}$, área de atuação, Pediatria, compararam qualidade de vida de 22 famílias de crianças com 3 e 7 anos de idade, que foram recém-nascidos com peso inferior a $1500 \mathrm{~g}$ e que apresentaram seqüelas neurológicas, 17 com paralisia cerebral e 5 com hidrocefalia e 15 outras famílias de crianças que foram recémnascidos com peso inferior a $1500 \mathrm{~g}$ e que não desenvolveram seqüelas neurológicas. Concluíram que o impacto da prematuridade e das seqüelas neurológicas nas famílias incidiu no relacionamento com as crianças, nos planos para o futuro das crianças e na vida pessoal dos pais. A família é mais afetada quando há um filho impossibilitado de andar, pela enorme demanda que este solicita em termos de tempo e recursos, causando prejuízos à vida social dos pais. A maioria dos pais, apesar dos problemas, é grata pela vida de seus filhos e pelos cuidados neonatais e de suporte terapêutico que receberam. O instrumento utilizado foi questionário estruturado com dados sobre memórias do período neonatal, estresse relacionado à comunicação da informação médica, comportamento dos profissionais e impacto financeiro. A análise foi realizada através de teste estatístico de distribuição de frequiência.

Hille et al. ${ }^{27}$, área de atuação Medicina Preventiva, compararam problemas de comportamento em 405 crianças entre 8 e 10 anos de idade que foram recém-nascidos com peso inferior a 1000g, em 4 países: Noruega, Alemanha, Canadá e Estados Unidos da América. Concluíram que, apesar das diferenças culturais, os problemas de comportamento observados, como dificuldades de pensamento, de atenção e problemas de comportamento social são semelhantes nos 4 países, sugerindo a contribuição do fator biológico para estes problemas. O método aplicado foi questionário estruturado com dados sobre comportamento ansi- 
oso, retraído, agressivo, delinqüente, problemas de pensamento e de atenção, aferidos com a utilização de escalas numéricas.

Saiga ${ }^{19}$, área de atuação Neonatologia, avaliou estado de saúde e qualidade de vida em saúde de 150 adolescentes, aos 14 anos de idade. Estes foram recém-nascidos extremo baixo peso e apresentavam, desde seqüelas moderadas até problemas complexos múltiplos. A avaliação foi realizada pela comparação do ponto de vista destes adolescentes, de seus pais e dos profissionais de saúde. A partir de alguns atributos citados pelos pais, foram criados dois escores de avaliação da qualidade de vida em saúde: os sistemas de classificação HU12 e HU13, usados para descrever o estado de saúde, como habilidade de funcionalidade da criança em cada uma das diversas áreas, simultaneamente. Os atributos foram: felicidade, ausência de dor, habilidades sensorial, física, de comunicação, de se cuidar e dificuldades de aprendizado e de escolaridade. Foram pré-selecionados 5 estados de saúde hipotéticos. Os adolescentes deveriam imaginar a si mesmos vivendo em cada um dos 5 estados de saúde pelos próximos 60 anos (auto percepção). Os pais deveriam imaginar seus filhos em cada um daqueles estados (percepção dos pais) e os profissionais de saúde deveriam imaginar seus paciente em cada um daqueles estados (percepção dos profissionais). Segundo a percepção dos profissionais, os adolescentes apresentaram grande prevalência de limitações de seu estado funcional. Os próprios adolescentes avaliaram-se num nível de qualidade de vida em saúde relativamente mais elevado, em relação à percepção dos profissionais. Os pais também avaliaram os filhos como tendo escores de qualidade de vida em saúde superiores aos correspondentes a suas seqüelas, quando considerada a percepção dos profissionais de saúde. Há aparente incongruência, do ponto de vista dos profissionais, entre a percepção dos pacientes sobre seu estado de saúde, bem estar e satisfa- ção com a vida, que é discordante com seu estado de saúde objetivo e com suas seqüelas. A qualidade de vida elevada, relatada pelos adolescentes e seus pais poderia ser causada por um ganho secundário, que ocorre quando indivíduos com impedimentos se adaptam a sua nova condição, reinterpretam suas vidas e reconstituem significados pessoais para seus papéis sociais: aprendem sobre suas dificuldades, têm controle sobre seus corpos e suas mentes e intensificam seu apreço pelos aspectos positivos da vida. A análise foi realizada através de teste quantitativo estatístico de distribuição de freqüência.

Hack et al. ${ }^{9}$, área de atuação, Neonatologia, estudaram 242 adolescentes aos 20 anos de idade que foram recém-nascidos com peso inferior a $1500 \mathrm{~g}$ avaliando nível de escolaridade e freqüência de doença crônica. Os resultados mostraram que, quando comparados a adolescentes que foram recém-nascidos a termo, os adolescentes que foram recém-nascidos extremo baixo peso apresentaram coeficiente de inteligência diminuído, nível de escolaridade mais baixo, traduzido por menor freqüência de graduação no ensino médio, e maior frequiência de doenças crônicas. Foi aplicado questionário estruturado e, como analise estatística, testes do qui-quadrado, regressão logística e linear múltipla.

Saigal et al..$^{28}$, área de atuação Neonatologia, compararam o impacto da prematuridade em famílias de adolescentes que foram recém-nascidos de extremo baixo peso, com um grupo controle. O objetivo foi avaliar a atitude de manter o tratamento ativo, procurando identificar se o impacto da prematuridade foi duradouro e se houve impacto crescente significativo nos pais dos adolescentes que apresentaram deficiências. Os resultados mostraram que, nos primeiros 6 meses após o nascimento, os pais apresentaram sofrimento significativo e grande preocupação pela saúde física e emocional de seus filhos com repercussões em suas 
saúdes emocionais e efeitos negativos na família. Relataram ter pouco tempo para si mesmos. Outros filhos foram afetados negativamente, talvez pelo menor tempo de atenção dos pais. Foi mais difícil obter trabalho, já que o recém-nascido prematuro exigiu maior cota de tempo para seu cuidado. A observação posterior mostrou que os pais dos adolescentes que foram recémnascidos com extremo baixo peso e que não apresentaram sequielas superaram os efeitos negativos da prematuridade. Os pais dos adolescentes com deficiências mantiveram impacto negativo. A longo prazo, todos os pais se ajustaram bem ao trabalho e à vida familiar. Apesar dos efeitos negativos, os pais foram favoráveis à manutenção do tratamento ativo a seus recém-nascidos no período em que estiveram internados em Unidade de Tratamento Intensivo Neonatal . Foi utilizado questionário com 23 itens sobre dados da vida profissional, conjugal e familiar dos pais, relacionados à prematuridade. A análise foi realizada através de testes estatísticos de distribuição de freqüência e quiquadrado.

Saigal et al..$^{29}$, área de atuação Neonatologia, Psicologia, realizaram estudo para avaliar a auto-estima de 132 adolescentes que foram recém-nascidos de extremo baixo peso comparando-os a 127 adolescentes que foram recém-nascidos de peso normal. Concluíram que, em geral, não há diferenças na auto-estima entre os dois grupos. O método empregado foi questionário estruturado. A análise foi realizada através de teste estatístico de covariância.

Os trabalhos para avaliação do impacto do nascimento de um recém-nascido prematuro extremo em sua família e, a longo prazo, o impacto sobre si mesmo, que utilizam métodos qualitativos, estão começando a surgir. Serão citados seis trabalhos:

Lamy ${ }^{11}$, área de atuação Neonatologia, estudou a vivência de pais de recém-nascidos submetidos a tratamento em Unidade de Tratamento Intensivo Neonatal, no período de inter- nação destas crianças. Concluiu que os pais apresentam confusão de sentimentos tais como culpa, medo e negação, o que dificulta o desenvolvimento do apego entre pais e filhos. As informações médicas não são bem compreendidas pois a comunicação entre pais e equipe de saúde, em geral, é difícil. Os pais têm sua vida pessoal e profissional devastadas, pela perspectiva de ter um filho com seqüelas e sob risco de morte. A autora utilizou, como métodos, entrevista aberta do tipo história de vida, definida como relato das experiências vividas por uma pessoa, um grupo ou uma organização e como os atores interpretam suas experiências e observação participante, definida como o contato direto do pesquisador com o fenômeno observado, com o objetivo de coletar, no contexto natural, as ações dos atores, partindo de sua perspectiva. O observador participa da interação em situações espontâneas e formais, interrogando sobre razões e significados. ${ }^{30-31}$ Análise dos dados foi realizada por princípios da hermenêutica, abordagem compreensiva de entender o outro, entender-se no outro, colocarse a si mesmo no lugar do outro para interpretar, estabelecer relações e extrair conclusões ${ }^{21}$.

$\mathrm{Gaíva}^{32}$, área de atuação Enfermagem, estudou a vida cotidiana de 18 crianças em idade escolar que nasceram com idade gestacional menor que 37 semanas e peso inferior a $2000 \mathrm{~g}$ e de suas famílias. Concluiu que o nascimento de um filho prematuro, para a família, é uma experiência difícil; o cuidar do filho envolve todos os membros da rede familiar e até a rede de vizinhos; a mãe assume o papel central em aspectos da socialização da criança, como a afetividade e, dentro do lar, esta criança é, muitas vezes, superprotegida. Foram utilizadas entrevista semi-estruturada, definida como perguntas fechadas (estruturadas) e abertas (o entrevistado tem a possibilidade de discorrer o tema proposto sem respostas ou condições préfixadas pelo pesquisador) e observação participante. A análise dos dados foi realizada pela 
técnica da análise temática, definida como a identificação dos núcleos de sentido que compõem uma comunicação, cuja presença ou freqüência signifiquem alguma coisa para o objeto analítico visado. $^{21}$

Nystrom e Axelsson $^{33}$, área de atuação Ciências da Saúde, estudaram a experiência materna de separação de seus recém-nascidos a termo que foram internados em Unidade de Tratamento Intensivo Neonatal. As mães relataram sentirem estar do "lado de fora", o que envolveu desespero, solidão e impotência, perda do controle, instabilidade emocional, culpa e insegurança e, em relação ao resultado do tratamento, confiança, alívio e amor. Foram utilizadas narrativas de 8 mães, narrativa definida como história coerente, com subtramas, organizada em sequiência cronológica, que apresenta disposição mais descritiva do que analítica e enfoque central que diz respeito ao homem e não às circunstâncias; trata do particular e do específico, de preferência ao coletivo e ao estatístico, Stone ${ }^{34}$. A análise foi realizada pela hermenêutica e pelo método fenomenológico, definido como a compreensão das estruturas de relevância dos grupos observados, respeitando a liberdade de seus componentes como atores sociais, com ênfase nos indivíduos enquanto agentes sociais, Minayo ${ }^{21}$.

Bialoskurski et al. ${ }^{35}$, área de atuação Enfermagem, avaliaram o processo da construção do apego entre a mãe e seu recém-nascido internado em Unidade de Tratamento Intensivo Neonatal. Concluíram que este processo não foi automático sendo os fatores significativos para sua construção: estado de saúde da mãe e do recém-nascido, circunstâncias do ambiente e qualidade do cuidado que o recém-nascido recebeu. Foi utilizada entrevista aberta, com dados qualitativos de observação da mãe no cuidado ao recém-nascido. Os dados foram analisados por comparação das narrativas registradas em notas de campo e em transcrições.

Cox e Bialoskurski ${ }^{36}$, área de atuação
Enfermagem, realizaram estudo sobre os fatores que facilitam o vínculo entre a mãe e a família, com o recém-nascido, em períodos de separação pós-parto prematuro. Concluíram que, quando a mãe e a família são apoiados através de auxílio individualizado e há comunicação adequada, o vínculo pode ser facilitado. Utilizaram três métodos qualitativos: observação participante, entrevista não estruturada e entrevista com grupos focais, definida como reunião de um pequeno grupo de informantes, escolhidos a partir de um determinado grupo, cujas idéias e opiniões são do interesse da pesquisa com a presença de um animador que intervém para focalizar e aprofundar a discussão. ${ }^{21}$ Fizeram uso, também, de um método quantitativo, questionário de Leske, que avalia as necessidades de famílias que têm recém-nascido sob cuidado intensivo.

Jackson et al. ${ }^{37}$, área de atuação Ciências da Saúde, Pediatria, realizaram estudo de como 7 mães e pais de recém-nascidos prematuros descrevem sua experiência de cuidar de seus filhos durante os primeiros 18 meses de vida das crianças. Concluíram que a internalização da parentalidade é um processo que dependente de tempo, da síntese das experiências de alienação, responsabilidade, confiança e familiaridade com a criança. A estruturação da parentalidade parece depender da expectativa dos pais com o "papel-de-serem-pais", da condição de saúde e do ambiente de cuidado da criança. O método utilizado foi entrevista e, para análise dos dados, o método fenomenólogico.

\section{COMENTÁRIOS}

Os estudos que abordam o impacto da prematuridade na família, em sua maioria, utilizam metodologia quantitativa e são realizados na área da Medicina, principalmente Neonatologia e Psiquiatria. Atualmente, a metodologia qualitativa é aplicada a estes estudos, principalmente, por profissionais de áreas não mé- 
dicas. São necessárias novas pesquisas para que os Médicos Neonatologistas conheçam a especificidade da metodologia qualitativa em gerar evidências podendo ser uma fonte de observações tão significativa quanto aquelas pro- venientes da pesquisa quantitativa, para estudos que abordam o impacto do nascimento de um recém-nascido prematuro extremo em sua família e, a longo prazo, o impacto sobre si mesmos.

\begin{abstract}
The objective of this study was to review how the literature focus impact of prematurity on the family and, at long-term, the impact on the child. We did so mainly focalizing the methodology used and the authors' area of work. The review was based on Medline and LILACS databases using the key-words: "premature, family"; "intensive care unit, neonatal"; "quality of life"; "qualitative research". Articles were selected from medical areas like Pediatrics, Psychiatry, Psychosocial Medicine and from other areas like Nursing and Human Sciences, published in the languages English, Spanish and Portuguese, within the last ten years and articles identified from the bibliographic references in the first articles. Postgraduate theses with most relevant information were gathered. Eleven articles were reviewed which employed quantitative methodology: structured and semi-structured interviews, approached by statistics tests like frequency distribution, chisquared, logistic regression, covariance, and six studies, two postgraduate theses and four articles which have used qualitative methodology like not-structured interview, focus group interview, life history and participant-observation, approached by phenomenology, phenomenologichermeneutic method and thematic analysis. It was concluded that mostly studies boarding the impact of prematurity on the family use quantitative methodology in the area of Medicine, mostly Neonatology and Psychiatry. Qualitative methodology begins to be applied to these studies, mainly by professionals from other areas that Medicine. New research is necessary for the physicians, specially for Neonatologists to learn the specificity of qualitative methodology in generating meaningful information for studies about prematurity and family.
\end{abstract}

Key-words: Premature. Family. Intensive care unit. Neonatal. Quality of life. Qualitative research.

\section{REFERÊNCIAS}

1. Green M. Parent care in intensive care unit. Am J Dis Child. 1979;133(11):1119-20.

2. O'Shea TM, Klinepeter KL, Goldstein DJ, Jackson BW, Dillard RG. Survival and developmental disability in infants with birth weights of 501 to 800 grams, born between 1979 and 1994. Pediatrics. 1997;100(6):982-6.

3. Saigal S, Stoskopf B, Feeny D, Furlong W, Burrows E, Rosenbaum P, et al. Differences in preferences for neonatal outcomes among health care professionals, parents, and adolescents. JAMA. 1999;281(21):1991-7.

4. Vohr BR, Wrigth LL, Dusick AM, Mele L, Verter J, Steichen JJ. Neurodevelopmental and functional outcomes of extremely low weigth infants in the National Institute of Child Health and Human Development Neonatal Research, 1993-1994. Pediatrics. 2000;105:1216-26.

5. McCormick MC. The outcomes of very low birth weight infants: are we asking the right questions? Pediatrics. 1997;99(6):869-76.

6. Rivers A, Caron B, Hack M. Experience of families with very low birthweight children with neurologic sequelae. Clin Pediatr (Phila). 1987;26(5):223-30.

7. Taylor HG, Klein N, Hack M. School-age consequences of birth weight less than 750g: a review and update. Dev Neuropsychol. 2000;17(3):289-321.

8. Msall ME Tremont MR. Functional outcomes in self-care, mobility, communication, and learning in extremely low-birth weight infants. Clin Perinatol. 2000;27(2):381-401.

9. Hack M, Flannery DJ, Schluchter M, Cartar L, Borawski E, Klein N. Outcomes in young adulthood for very-low-birth-weight infants. N Engl J Med. 2002;346(3):149-57.

10. Klaus MH, Kennell JH. Pais/Bebês: a formação do apego. $\mathbf{2}^{\mathrm{a}}$ ed. Porto Alegre: Artes Médicas; 1993.

11. Lamy ZC. Estudo das situações vivenciadas por pais de recém-nascidos internados em unidades 
de terapia intensiva neonatal [dissertação]. Rio de Janeiro: Instituto Fernandes Figueira da Fundação Oswaldo Cruz; 1995.

12. Javorski M. Os significados do aleitamento materno para mães de prematuros em cuidado canguru [dissertação]. Ribeirão Preto: Escola de Enfermagem de Ribeirão Preto da Universidade de São Paulo; 1997.

13. Videla M. O bebê de alto risco: intervenções multidisciplinares. Anais do $8^{\circ}$ Congresso Nacional da ABENEPI; 1987; Belo Horizonte. Belo Horizonte: ABENEPI; 1987.. p.106

14. Preyde M, Ardal F. Effectiveness of a parent "buddy" program for mothers of very preterm infants in a neonatal intensive care unit. CMAJ. 2003;168(8):969-73.

15. Zeanah $\mathrm{CH}, \mathrm{McD}$ onough $\mathrm{S}$. Clinical approaches to families in early intervention. Semin Perinatol. 1989;13(6):513-22.

16. Ritchie SK. Primary care of the premature infant discharged from the neonatal intensive care unit. MCN Am J Matern Child Nurs. 2002;27(2):76-85.

17. Bowlby J. Cuidados maternos e saúde mental. $2^{\mathrm{a}}$ ed. São Paulo: Martins Fontes; 1988.

18. Raddish M, Merritt TA. Early discharge of premature infants: a critical analysis. Clin Perinatol. 1998;25(2):499-520.

19. Saigal S. Perception on health status and quality of life of extremely low birthweight survivors: the consumer, the provider, and the child. Clin Perinatol. 2000;27(2):403-19.

20. Minayo MCS, Sanches O. Quantitativoqualitativo: oposição ou complementaridade? Cad Saúde Pública. 1993;9(3):237-48.

21. Minayo MCS. O desafio do conhecimento: pesquisa qualitativa em saúde. $3^{\mathrm{a}}$ ed. São Paulo: Hucitec-Abrasco; 1992.

22. Streiner DL, Saigal S, Burrows E, Stoskopf Rosenbaum P. Attitudes of parents and health care professionals toward active treatment of extremely premature infants. Pediatrics. 2001;108(1):152-7.

23. Holditch-Davis D, Bartlett TR, Blickman AL, Miles MS. Posttraumatic stress symptoms in mothers of premature infants. J Obstet Gynecol Neonatal Nurs. 2003;32(2):161-71.

24. Tommiska V, Ostberg M, Fellman V. Parenteral stress in familiares of 2 year old extremely low birthweight. Arch Dis Child Fetal Neonatal Ed. 2002;86(3):161-4.

25. Singer LT, Salvator A, Guo S, Collin M, Lilien L, Baley J. Maternal psycohological distress and parenting stress after the birth of a very low-birthweigth infant. JAMA. 1999;281(9):799-805.

26. Pierrehumbert B, Nicole A, Muller-Nix C, ForcadaGuex M, Ansermet F. Parenteral post-traumatic reactions after premature birth: implications for slleping and eating problems in the infant. Arch Dis child Fetal Neonatal Ed. 2003;88(5):400-4.

27. Hille ET, Ouden AL, Saigal S, Wolke D, Lambert $\mathrm{M}$, Whitaker A, et al. Behavioural problems in children who weigh $1000 \mathrm{~g}$ or less at birth in four countries. Lancet. 2001;357(9269):1641-3.

28. Saigal S, Burrows E, Stoskopf BL, Rosenbaum PL, Streiner D. Impact of extreme prematurity on families of adolescent children. J Pediatr. 2000;137(5):701-6.

29. Saigal S, Lambert M, Russ C, Hoult L. Self-esteem of adolescents who were born prematurely. Pediatrics. 2002;109(3):429-33.

30. Denzin NK. The research act: a theoretical introduction to sociological methods. Englewood Cliffs (NJ): Prentice-Hall; 1989.

31. Chizzotti A. Pesquisa em ciências humanas e sociais. $4^{\mathrm{a}}$ ed. São Paulo: Cortez; 2000.

32. Gaiva MAM. Cotidiano de escolares nascidos prematuros: percepção de crianças e familiares [dissertação]. São Paulo: Escola de Enfermagem de Ribeirão Preto da Universidade de São Paulo; 1997.

33. Nystrom K, Axelsson K. Mother's experience of being separated from their newborns. J Obstet Gynecol Neonatal Nurs. 2002;31(3):275-82.

34. Stone L. O ressurgimento da narrativa: reflexões sobre uma nova velha história. Rev História Univ Campinas. 1991;213:13-46.

35. Bialoskurski M, Cox CL, Hayes JA. The nature of attachment in a neonatal intensive care unit. $\mathrm{J}$ Perinat Neonatal Nurs. 1999;13(1):66-77.

36. Cox C, Bialoskurski M. Neonatal intensive care: communication and attachment. Br J Nurs. 2001;10(10):668-76.

37.Jackson K, Ternestedt BM, Schollin J. From alienation to familiarity: experiences of mothers and fathers of preterm infants. $J$ Adv Nurs. 2003;43(2):120-9. 